\title{
Análise da Curvatura de Modulações Não-Lineares Associadas a Curvas
}

\author{
Rodrigo Gusmão Cavalcante e Reginaldo Palazzo Jr.
}

\begin{abstract}
Resumo-Neste trabalho usamos uma interpretação geométrica que associa esquemas de modulação a curvas no espaço Euclidiano para construir e analisar novas modulações nãolineares. Com isso, foi possível verificar que além do comprimento da curva, a curvatura é um outro parâmetro de grande relevância no projeto de modulações não-lineares, pois influencia diretamente em seu desempenho, mais precisamente no valor do erro quadrático médio. Com o objetivo de minimizar a ocorrência de erro de limiar uma condição entre a energia média de ruído e a curvatura da modulação foi determinada. Além disso, uma modulação não-linear foi proposta e seu desempenho foi comparada ao desempenho da modulação associada à curva espiral de Arquimedes. Neste caso, a influência da curvatura no desempenho de modulações não-lineares foi evidenciada.
\end{abstract}

Palavras-Chave-Modulação não-linear, curvatura, erro quadrático médio, análise de desempenho.

Abstract-In this paper we use a geometrical interpretation which associates modulation schemes to curves in Euclidean space to design and analyze new twisted modulations. From this, it was possible to verify that besides the length of a curve, the curvature is another parameter of great relevance in the design of nonlinear modulations, since this parameter affects the performance under the mean-square error criterion. In order to minimize the threshold error a condition had to be established involving the noise average energy and the curvature of the modulation. In addition to this, a non-linear modulation is proposed and its performance is compared with the performance of a modulation making use of the Archimedean spiral curve. In this case the influence of the curvature in the performance of non-linear modulations is brought to light.

Keywords - Twisted modulation, curvature, mean-square error, performance analysis.

\section{INTRODUÇÃO}

Neste trabalho considere o sistema de comunicações apresentado de forma simplificada na Figura 1. Neste sistema a variável aleatória $m$, com função densidade de probabilidade a priori $p_{m}$, é transmitida pelo sinal modulado $s_{m}(t)$ através de um canal com ruído aditivo, $n(t)$. Além disso, após a observação do sinal recebido, $r(t)$, o receptor estima uma saída $\widehat{m}$, de $m$.

Em [3], as técnicas de modulação foram associadas a curvas no $\mathbb{R}^{N}$. Tal interpretação geométrica possibilitou em [7] e [8] o projeto e a análise de desempenho de modulações usando algumas ferramentas da geometria diferencial [5] e, mais geralmente, da geometria Riemanniana [6].

A interpretação geométrica para a técnica de modulação proposta em [3] é baseada na possibilidade do sinal modulado

Rodrigo Gusmão Cavalcante e Reginaldo Palazzo Jr., Departamento de Telemática (DT), Faculdade de Engenharia Elétrica e de Computação (FEEC), Universidade Estadual de Campinas (UNICAMP), emails: rgc@dt.fee.unicamp.bre palazzo@dt.fee.unicamp.br.

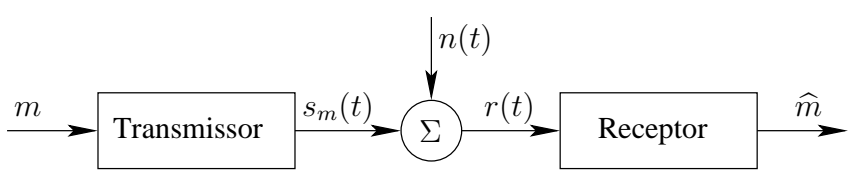

Fig. 1. Exemplo de um diagrama de blocos de sistema de comunicações para a transmissão de uma variável aleatória contínua.

$s_{m}(t)$ ser decomposto em uma base de sinais ortogonais de energia unitária, $\varphi_{i}(t), i=1, \ldots, N$, da seguinte forma

$$
s_{m}(t)=s_{1}(m) \varphi_{1}(t)+s_{2}(m) \varphi_{2}(t)+\cdots+s_{N}(m) \varphi_{N}(t) .
$$

Neste caso, as portadoras $\varphi_{i}(t)$ podem ser interpretadas como um conjunto de vetores ortonormais $\left\{\boldsymbol{\varphi}_{i}\right\}$, gerando uma base para o $\mathbb{R}^{N}$. Com isso, o sinal modulado $s_{m}(t)$ pode ser representado nessa base vetorial da seguinte maneira

$$
\begin{aligned}
\mathbf{s}_{m} & =s_{1}(m) \boldsymbol{\varphi}_{1}+s_{2}(m) \boldsymbol{\varphi}_{2}+\cdots+s_{N}(m) \boldsymbol{\varphi}_{N} \\
& =\left[s_{1}(m), s_{2}(m), \ldots, s_{N}(m)\right] .
\end{aligned}
$$

Isto é, o sinal modulado $s_{m}(t)$, representado vetorialmente por $\mathbf{s}_{m}$, pode ser interpretado como uma curva parametrizada $\mathbf{s}_{m}: I \in \mathbb{R} \rightarrow \mathbb{R}^{N}, m \in I$.

Neste trabalho, o parâmetro considerado para medir o desempenho do sistema de comunicações da Figura 1 é o erro quadrático médio, definido como

$$
\overline{\epsilon^{2}} \triangleq \mathrm{E}\left[(m-\widehat{m})^{2}\right]=\overline{(m-\widehat{m})^{2}},
$$

onde $\mathrm{E}[\cdot]$ é o operador esperança, medido conjuntamente para todas as $m$ entradas permitidas e todas as possíveis estimativas $\hat{m}$ determinadas pelo receptor após a ação do ruído.

Além disso, para um melhor entendimento dos resultados descritos neste trabalho, os desempenhos das modulações propostas foram apresentados em função de gráficos da relação sinal ruído do canal de transmissão (CSNR) versus a relação sinal ruído da informação transmitida (SNR), definidos como

$$
\mathrm{CSNR} \triangleq \frac{\bar{E}_{m}}{\mathcal{N}} \quad \text { e } \quad \mathrm{SNR} \triangleq \frac{\overline{m^{2}}}{\overline{\epsilon^{2}}}
$$

onde $\overline{m^{2}}$ é a energia média (ou variância) da variável aleatória $m$ de média nula, $\mathcal{N}$ é a energia média de ruído presente no canal de transmissão e $\bar{E}_{m}$ é a energia média de transmissão do sinal $s_{m}(t)$, definida como

$$
\bar{E}_{m} \triangleq \mathrm{E}\left[\left|\mathbf{s}_{m}\right|^{2}\right]=\mathrm{E}\left[\int_{-\infty}^{\infty} s_{m}^{2}(t) d t\right],
$$

onde o operador esperança, neste caso, é medido para todos 
os possíveis valores de $m$ e $|\cdot|$ é a norma de um vetor.

Neste trabalho, as curvas de desempenho (CSNR versus SNR) dos sistemas propostos foram comparadas à curva de um sistema ótimo, denotada pela sigla OPTA e definida em [2] pela seguinte relação

$$
\mathrm{SNR}=(1+\mathrm{CSNR})^{N / M},
$$

onde $(N / M)$ é a razão entre as dimensões dos sinais modulado e não modulado, respectivamente. Por exemplo, para o caso particular de modulações associadas a curvas planares essa razão vale 2 , pois neste caso $\mathbf{s}_{m} \subset \mathbb{R}^{N=2}$ e $m \in \mathbb{R}^{M=1}$.

Em [3] a interpretação geométrica que associa modulações a curvas no $\mathbb{R}^{N}$ foi utilizada para verificar que o erro quadrático médio é inversamente proporcional ao quadrado do comprimento de arco da curva associada à modulação. Tal fato é válido quando a energia média do ruído é suficientemente pequena, de maneira que $\mathbf{s}_{m}$ possa ser aproximado, com o auxílio da série de Taylor, da seguinte forma

$$
\mathbf{s}_{m} \approx \mathbf{s}_{0}+\left(m-m_{0}\right) \mathbf{s}_{0}^{\prime}, \quad \text { com } \quad \mathbf{s}_{0}^{\prime}=\left.\frac{d \mathbf{s}_{m}}{d m}\right|_{m=m_{0}},
$$

onde $\mathbf{s}_{0}^{\prime}$ é a derivada de $\mathbf{s}_{m}$ em relação a $m$ no ponto $m_{0}$.

A Figura 2 ilustra a recepção por máxima verossimilhança (ML) de uma modulação não-linear usando essa aproximação quando $\mathbf{s}_{0}$ é transmitido e o ruído é AWGN. Nesse caso, o receptor obtém a estimativa $\hat{m}$ pela minimização de $\left|\mathbf{r}-\mathbf{s}_{m}\right|$, isto é, projetando o sinal recebido $\mathbf{r}$ na direção da reta tangente à curva $\mathbf{s}_{m}$ em $\mathbf{s}_{0}$, equação (7).

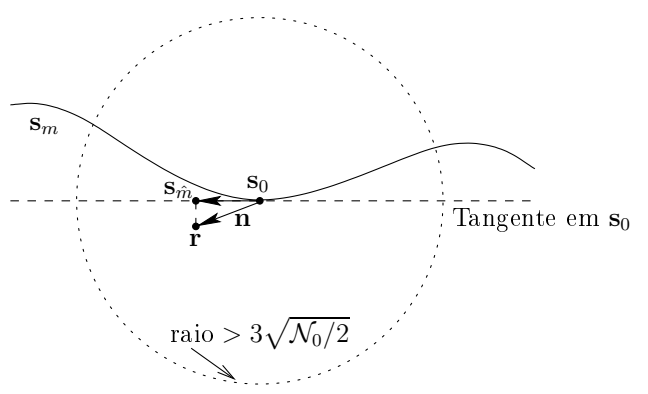

Fig. 2. Recepção por máxima verossimilhança de um sistema de comunicações usando uma modulação não-linear quando o ruído de transmissão é AWGN e sua energia média é suficientemente pequena.

Neste contexto, a relação verificada em [3] para o erro quadrático médio dado que $m_{0}$ foi enviado é dada por

$$
\overline{\epsilon^{2}}\left(m_{0}\right)=\frac{\mathcal{N}_{0} / 2}{\left|\mathbf{s}_{0}^{\prime}\right|^{2}}=\frac{\mathcal{N}_{0} / 2}{S^{2}},
$$

onde o parâmetro $S$ é chamado de fator de expansão da modulação. Geometricamente, o fator de expansão está associado ao comprimento da curva associada a modulação, isto é,

$$
L=\int_{-1}^{1}\left|\mathbf{s}_{m}^{\prime}\right| d m
$$

que é igual a $2 S$ caso o fator de expansão seja uniforme (constante).

Este trabalho está organizado da seguinte forma. Na Seção II determinamos que o valor da curvatura de uma modulação influencia no desempenho do sistema de comunicações apresentado na Figura 1. Na Seção III propomos um novo esquema de modulação não-linear e seu desempenho foi analisado e comparado ao desempenho da modulação associada à curva espiral de Arquimedes. Nesta caso, a influência da curvatura no desempenho da modulação proposta foi evidenciada. Finalmente, na Seção IV as conclusões são apresentadas.

\section{INFLUÊNCIA DA CURVATURA NO PROJETO DE MODULAÇÕES}

Sabe-se que a curvatura está associada à variação do vetor tangente de uma curva, então a curvatura de uma modulação linear é nula. Além disso, o uso de modulações não-lineares demonstra ser uma alternativa para aumentar o desempenho dos sistemas de comunicações. Portanto, como a curva associada a uma modulação não-linear possui curvatura diferente de zero, então a existência de curvatura pode influenciar no desempenho do sistema de comunicação.

Contudo, antes de quantificar a influência da curvatura no projeto de modulações é necessário introduzir o conceito de erro de limiar (threshold) definido em [3]. Para tanto, inicialmente considere a modulação linear apresentada na Figura 3(a), com $\mathbf{s}_{0}$ transmitido e $\mathbf{r}$ recebido. A Figura 3(b) exemplifica o processo de demodulação usando um receptor ML considerando que o sinal $\mathbf{r}$ pertença a um dos círculos $c_{1}, c_{2}$ ou $c_{3}$, que caracterizam a região de ação de um ruído AWGN com diferentes valores de energia média. Neste caso, note que o receptor determina três regiões para os possíveis valores de $\hat{m}$, sendo cada uma delas associada a um dos círculos considerados.

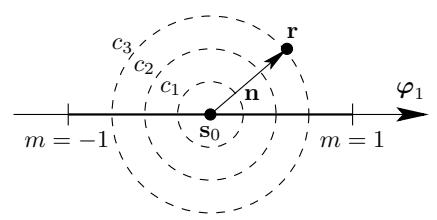

(a) Exemplo na modulação linear.

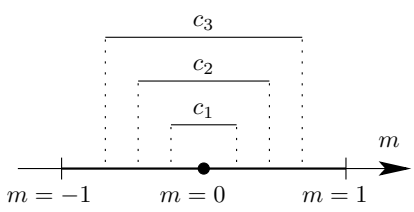

(b) Possíveis valores para $\hat{m}$.
Fig. 3. Interpretação geométrica do erro de limiar em uma modulação linear sob a ação de um ruído AWGN.

Em seguida, considere a modulação não-linear ilustrada na Figura 4(a), também com $\mathbf{s}_{0}$ transmitido e $\mathbf{r}$ recebido. Nesta modulação, a ação do ruído pode caracterizar uma região de limiar de decisão, Figura4(b), bem diferente da região de limiar de uma modulação linear, Figura 3(b). Este fato implica que sinais modulados sobre curvas com grande comprimento (menor $\overline{\epsilon^{2}}$ ), podem possuir sinais próximos em termos da distância Euclidiana e um novo tipo de erro pode existir na recepção, o erro de limiar, causando uma espécie de "confusão" no receptor, como ilustra a região de decisão da Figura 4(b). Além disso, devemos ressaltar que tal erro é certamente o principal responsável por impedir que o comprimento da curva seja aumentado indefinidamente com o objetivo de melhorar o desempenho da modulação.

Com isso, é interessante observar que para a modulação linear não há erro de limiar. Este fato será associado a seguir ao valor da curvatura da curva associada a essa modulação, 


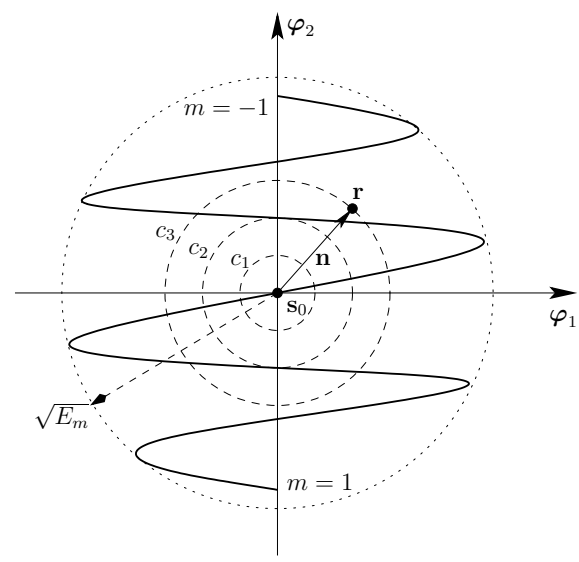

(a) Exemplo na modulação não-linear.

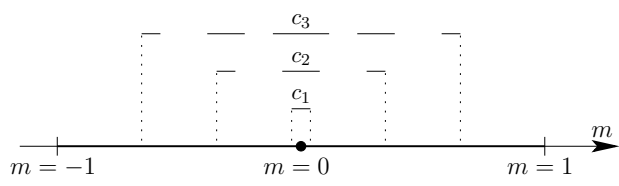

(b) Possíveis valores para $\hat{m}$.

Fig. 4. Interpretação geométrica do erro de limiar em uma modulação nãolinear sob a ação de um ruído AWGN.

que no caso é nula. Além disso, quando o ruído for AWGN e o receptor for ML, então o erro de limiar é influenciado pela distância Euclidiana entre os sinais.

Para medir a influência da curvatura no projeto de modulações considere que a Figura 5(a) represente uma modulação linear e a Figura 5(b) uma modulação não-linear, ambas sob a ação de um ruído AWGN. Além disso, considere que os círculos apresentados nessas figuras representem a região de ação do ruído dado que cada um dos sinais $\mathbf{s}_{m_{i-1}}, \mathbf{s}_{m_{i}} \mathrm{e}$ $\mathbf{s}_{m_{i+1}}$, onde $m_{i-1}=m_{i}-\Delta m$ e $m_{i+1}=m_{i}+\Delta m$, sejam transmitidos. Assuma também que o fator de expansão é uniforme em ambas as modulações, o que implica que a diferença de comprimento entre os sinais é igual a $\Delta s=$ $S \Delta m$.

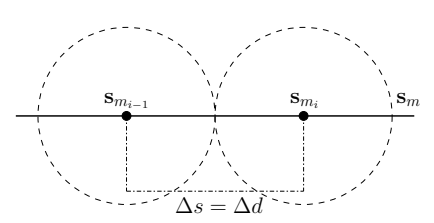

(a) Modulação linear.

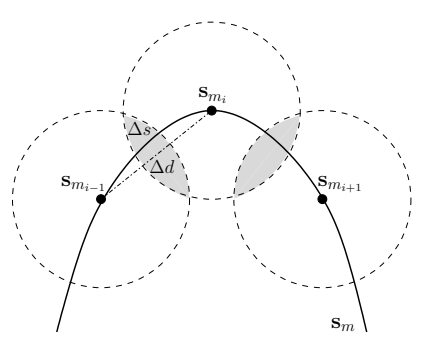

(b) Modulação não-linear.
Fig. 5. Análise da influência da curvatura de $\mathbf{s}_{m}$ no erro de limiar.

Portanto, fazendo uso de tais considerações, é possível realizar uma comparação geométrica entre essas duas modulações e verificar que a distância Euclidiana $(\Delta d)$ entre os sinais da modulação não-linear é, em geral, menor do que a distância Euclidiana na modulação linear. Tal idéia pode ser melhor compreendida verificando que na modulação não-linear existe uma região de interseção entre os círculos, fato que não ocorre na modulação linear, ou simplesmente notando que $\Delta d<\Delta \mathbf{s}$ na Figura 5(b). A seguir será mostrado que o fado de $\Delta d$ é menor que $\Delta \mathbf{s}$ na modulação não-linear por causa do valor de sua curvatura.

A curvatura depende da parametrização da curva associada a modulação. Se a curva for parametrizada pelo comprimento de $\operatorname{arco}$ (p.p.c.a), $\left|\mathbf{s}_{m}^{\prime}\right|=1$, então

$$
k=\left|\mathbf{s}_{m}^{\prime \prime}\right|
$$

caso contrário

$$
k=\frac{s_{1}^{\prime} s_{2}^{\prime \prime}-s_{2}^{\prime} s_{1}^{\prime \prime}}{\left[\left(s_{1}^{\prime}\right)^{2}+\left(s_{2}^{\prime}\right)^{2}\right]^{(3 / 2)}} .
$$

Para mostrar a influência da curvatura no desempenho das técnicas de modulação, considere a aproximação para $\mathbf{s}_{m}$ dada em [6], com fator de expansão uniforme e expressa em função de sua curvatura, dada por

$$
\mathbf{s}_{m}=\mathbf{s}_{0}+\left[\Delta s-\frac{k^{2}}{6}(\Delta s)^{3}, \frac{k}{2}(\Delta s)^{2}+\frac{k^{\prime}}{6}(\Delta s)^{3}\right],
$$

onde $\Delta s=S \Delta m$. Como o interesse é medir o quanto a distância Euclidiana entre os sinais diminui de uma modulação não-linear para uma modulação linear, a equação (11) foi usada com $\Delta d=\left|\mathbf{s}_{m}-\mathbf{s}_{0}\right|$, para obter a seguinte relação

$$
\frac{\Delta d}{\Delta s}=\sqrt{1-\frac{k^{2}}{12}(\Delta s)^{2}+\frac{k k^{\prime}}{6}(\Delta s)^{3}+\frac{\left(k^{\prime}\right)^{2}+k^{4}}{36}(\Delta s)^{4}} .
$$

Portanto, além da verificação geométrica realizada com o auxílio da Figura 5, é provado matematicamente a partir de (12) que se a curvatura for zero, então $\Delta d=\Delta s$. Contudo, se a curvatura for diferente de zero, então localmente $\Delta d<\Delta s$, para $\Delta s$ suficientemente pequeno. Neste caso, quanto menor for o valor de $\Delta d / \Delta s$ mais provável será a ocorrência de erro de limiar. Portanto, a curvatura influencia no desempenho dos sistemas de comunicações considerados.

Contudo, a perda de desempenho decorrente da existência de erros de limiar, geralmente, é compensada pela diminuição da energia média ou do aumento do fator de expansão propiciado pelo uso de uma modulação não-linear.

Com o intuito de diminuir a ocorrência de erro de limiar, a seguir será determinado um limitante superior para o valor da curvatura em função da energia média do ruído. Para tanto, considere um receptor de máxima verossimilhança, no qual $\hat{m}$ é um ponto de mínimo local de $\left|\mathbf{r}-\mathbf{s}_{m}\right|$ se satisfaz

$$
\left|\mathbf{s}_{\hat{m}}^{\prime}\right|^{2}-\left(\mathbf{r}-\mathbf{s}_{\hat{m}}\right) \cdot \mathbf{s}_{\hat{m}}^{\prime \prime}>0, \quad \text { quando } \quad\left(\mathbf{r}-\mathbf{s}_{\hat{m}}\right) \cdot \mathbf{s}_{\hat{m}}^{\prime}=0
$$

Com isso, usando a aproximação (7) e a expressão (10b), o fato do ruído (n) ser igual ao sinal recebido (r) menos o sinal transmitido $\left(\mathbf{s}_{0}\right)$ e considerando que a modulação possua fator de expansão uniforme, isto é $\mathbf{s}_{m}^{\prime} \cdot \mathbf{s}_{m}^{\prime \prime}=0$, então a condição (13) pode ser reescrita como

$$
\begin{gathered}
1-\frac{\left|\mathbf{s}_{\hat{m}}^{\prime \prime}\right|}{\left|\mathbf{s}_{\hat{m}}^{\prime}\right|^{2}}\left(\mathbf{n} \cdot \frac{\mathbf{s}_{\hat{m}}^{\prime \prime}}{\left|\mathbf{s}_{\hat{m}}^{\prime \prime}\right|}\right)>0 \Rightarrow 1-|k|\left(\mathbf{n} \cdot \frac{\mathbf{s}_{\hat{m}}^{\prime \prime}}{\left|\mathbf{s}_{\hat{m}}^{\prime \prime}\right|}\right)>0 \Rightarrow \\
\frac{\mathcal{N}_{0}}{2}<\frac{1}{|k|^{2}} \Rightarrow \frac{\mathcal{N}_{0}}{2} \leq \min _{m \in(-1,1)}\left\{\frac{1}{k^{2}}\right\},
\end{gathered}
$$

onde $|k|=\left|\mathbf{s}_{\hat{m}}^{\prime \prime}\right| /\left|\mathbf{s}_{\hat{m}}^{\prime}\right|^{2}$ e $\mathbf{n} \cdot \mathbf{s}_{\hat{m}}^{\prime \prime} /\left|\mathbf{s}_{\hat{m}}^{\prime \prime}\right|$ é a projeção do ruído AWGN em uma direção (dimensão), cuja energia média de 
ruído é igual a $\mathcal{N}_{0} / 2$.

Dessa forma, pode-se usar (14) para projetar geometricamente uma modulação com tolerância satisfatória ao erro de limiar, caso o quadrado do raio de curvatura seja sempre menor ou igual a energia média de ruído por dimensão $\left(\mathcal{N}_{0} / 2\right)$.

\section{MOdULAÇÃO SENOIDAL}

A modulação não-linear apresentada a seguir, chamada de modulação senoidal, foi projetada geometricamente com o auxílio de (2), (8) e (14). Para que o desempenho dessa modulação seja satisfatório foi considerado que a curva associada a essa modulação apresentasse algumas características geométricas importantes, tais como: um comprimento elevado para diminuir o erro quadrático médio, não possuir autointerseção e ter uma curvatura pequena para minimizar as ocorrências de erros de limiar. Além disso, a curva associada à modulação foi restrita a um círculo para que a energia média de transmissão pudesse ser reduzida.

Com isso, determinamos empiricamente que a curva associada à modulação senoidal possui parametrização dada por

$$
\mathbf{s}_{m}=A\left[m, \sqrt{1-m^{2}} \sin ^{\gamma}(\beta m)\right], \quad m \in(-1,1),
$$

onde $\beta \in \mathbb{R}$ e $\gamma \in\{1,1 / 3,1 / 5,1 / 7, \ldots\}$ são constantes desse esquema de modulação que influenciam em seu desempenho. A Figura 6 apresenta algumas dessas curvas para $\beta=3 \pi, 4 \pi, 5 \pi$ e $\gamma=1$. Neste caso, observe que essas curvas são senoides de diferentes freqüências e amplitude limitada por um círculo de raio $A$. Por esse motivo, a modulação em questão foi denominada de modulação senoidal.

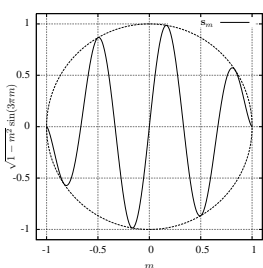

(a) $\beta=3 \pi$.

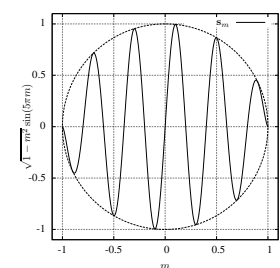

(b) $\beta=4 \pi$.

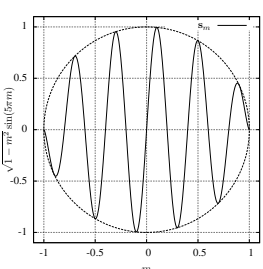

(c) $\beta=5 \pi$.
Fig. 6. Representação geométrica da modulação senoidal, para $\beta=3 \pi, 4 \pi$ e $5 \pi, \gamma=1$ e $A=1$.

A Figura 7 apresenta algumas outras curvas associadas à modulação senoidal calculadas usando a parametrização (15) para $\beta=3 \pi, 4 \pi, 5 \pi$ e $\gamma=1 / 7$. Comparando a Figura 7 com a Figura 6, pode-se observar que as curvas $\operatorname{com} \gamma=1 / 7$ apresentam maior comprimento e menor curvatura que as curvas com $\gamma=1$. Tais fatos são importantes para explicar a diferença de desempenho entre essas modulações usando as expressões (8) e (14).

No decorrer da análise de desempenho da modulação senoidal, as constantes $\beta$ e $\gamma$ serão associadas à largura de banda de transmissão, ao erro quadrático médio e ao erro de limiar. Contudo, primeiramente, é interessante notar de (15) que quando $\beta=0$, essa modulação é semelhante a modulação linear (a curva é uma reta) e quando $\gamma=0$, essa modulação é semelhante a modulação PM, cuja curva associada é um círculo.

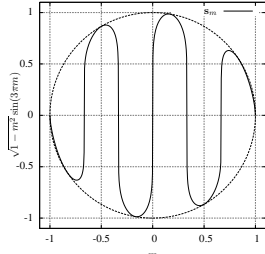

(a) $\beta=3 \pi$.

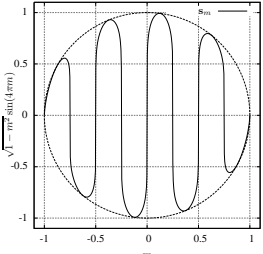

(b) $\beta=4 \pi$.

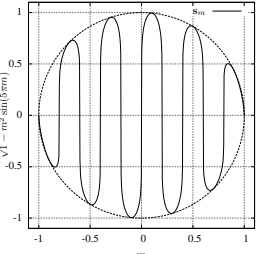

(c) $\beta=5 \pi$.
Fig. 7. Representação geométrica para a modulação senoidal, quando $\beta=$ $3 \pi, 4 \pi$ e $5 \pi, \gamma=1 / 7$ e $A=1$.

Além disso, observe que geometricamente o valor de $\beta$ indica a quantidade de "picos" da senoide. Conseqüentemente, quanto maior for o valor de $\beta$ maior será o comprimento da respectiva curva. Com isso, o erro quadrático médio de um sistema de comunicações usando essa modulação pode ser diminuído com o aumento de $\beta$, pois $\overline{\epsilon^{2}}$ é inversamente proporcional ao quadrado do fator de expansão da curva, como indica a equação (8). Contudo, caso o valor de $A$ seja mantido constante e o valor de $\beta$ seja aumentado, então a distância entre dois ramos consecutivos da senoide certamente irá diminuir $(d=\pi A / \beta)$, aumentando assim a ocorrência de erro de limiar. Tal fato pode ser melhor compreendido com o auxílio da Figura 8 que representa o envio de $m_{0}=1 / 3$ em um canal ruidoso, cujo raio de ação do ruído é caracterizado pelo círculo tracejado da Figura 8(a). Neste caso, o sinal enviado possui grande probabilidade de ser estimado por $\hat{m}=0$, $\hat{m}=1 / 3$ ou $\hat{m}=2 / 3$, como sugere $p\left(\hat{m} \mid m_{0}=1 / 3\right)$ na Figura 8(b).

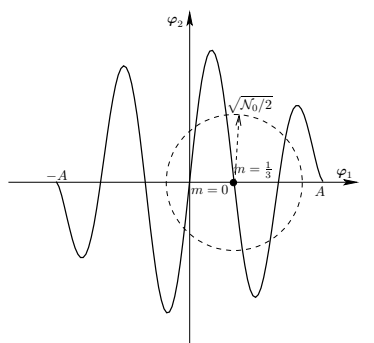

(a) $\mathbf{s}_{m} \operatorname{com} m_{0}=1 / 3$.

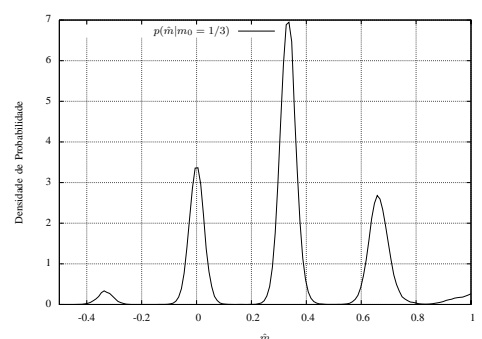

(b) $p\left(\hat{m} \mid m_{0}=1 / 3\right)$ e $\overline{\epsilon^{2}}=0.0703$.
Fig. 8. Análise de erro de limiar para uma modulação senoidal quando $\beta=3 \pi, \gamma=1, \mathcal{N}_{0}=A^{2} / 2$ e $m_{0}=1 / 3$.

Para efeito de comparação da largura de banda de transmissão da modulação senoidal com a largura de banda de outras modulações, considere o sinal modulante de uma única freqüência definido por

$$
m(t)=A_{m} \cos \left(2 \pi f_{m} t\right),
$$

onde $A_{m}$ é a amplitude do sinal modulante, que no nosso caso vale 1 , pois foi considerado que $m \in(-1,1)$. Neste caso, o sinal modulado usando a modulação senoidal é dado por

$$
\begin{aligned}
s_{m}(t)= & A \cos \left(2 \pi f_{m} t\right) \varphi_{1}(t)+A\left|\sin \left(2 \pi f_{m} t\right)\right| \\
& \times \sin ^{\gamma}\left(\beta \cos \left(2 \pi f_{m} t\right)\right) \varphi_{2}(t) .
\end{aligned}
$$

onde $\varphi_{1}(t)=\cos \left(2 \pi f_{c} t\right)$ e $\varphi_{2}(t)=\sin \left(2 \pi f_{c} t\right)$ são portadoras do transmissor. 


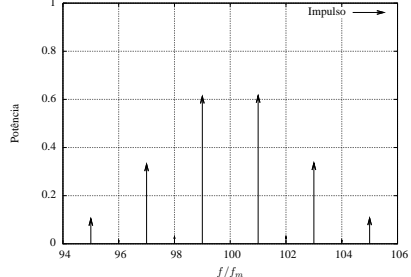

(a) $\beta=\pi$ e $B_{t}=10 f_{m}$.

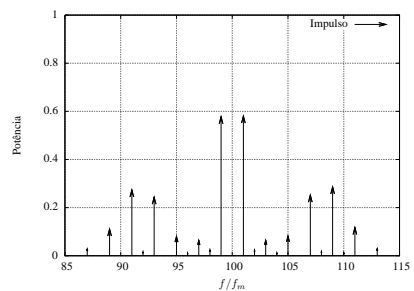

(c) $\beta=3 \pi$ e $B_{t}=26 f_{m}$.

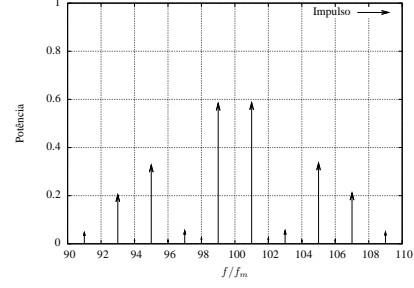

(b) $\beta=2 \pi$ e $B_{t}=18 f_{m}$.

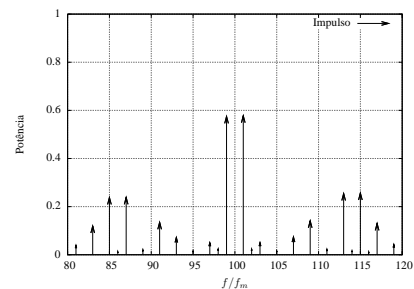

(d) $\beta=5 \pi$ e $B_{t}=38 f_{m}$.
Fig. 9. Espectros de amplitudes discretas de um sinal associado à modulação senoidal, normalizados em relação à amplitude $A$, para o caso do sinal modulante senoidal de freqüência fixa $f_{m}$. São mostrados somente os espectros correspondentes às freqüências positivas.

Com o auxílio da transformada discreta de Fourier foi realizada uma simulação computacional para obter a análise espectral do sinal (17) considerando $f_{c}=100 f_{m}$ e $\gamma=1$, veja Figura 9. Note que, o valor de $\beta$ altera a largura de banda de transmissão da modulação senoidal. Além disso, para um mesmo valor de $\beta$ a largura de banda dessa modulação é aproximadamente igual a largura de banda da modulação em freqüência (FM), veja [10].

Deve-se chamar a atenção para o fato dessa análise ter sido apresentada apenas para $\gamma=1$, porém foi verificado que quando este valor diminui, então o valor da largura de banda de transmissão aumenta. Entretanto, quando a análise espectral é realizada considerando um fator de expansão uniforme, então o parâmetro $\gamma$ não influencia tanto no cálculo de $B_{t}$.

Para realizar uma análise de desempenho da modulação senoidal, é necessário determinar o fator de expansão dessa modulação, pois de acordo com 8 o erro quadrático médio é inversamente proporcional ao quadrado de seu valor.

$$
S^{2}=1+\sin (\beta m)^{2 \gamma}\left(-\frac{m}{\sqrt{1-m^{2}}}+\frac{\gamma \beta \sqrt{1-m^{2}}}{\tan (\beta m)}\right)^{2} \text {. }
$$

A análise de desempenho dessa modulação foi realizada considerando um fator de expansão uniforme, porém não apresentamos neste trabalho uma reparametrização para (15) nesta condição ( $S$ constante), então alguns valores de $S=L / 2$ foram calculados e apresentados na Tabela I com o auxílio de (9), para $\beta=\pi, 3 \pi, \ldots, 9 \pi$ e $\gamma=1$ e $1 / 7$.

\section{TABELA I}

ALGUNS VALORES PARA O FATOR DE EXPANSÃO UNIFORME DA MODULAÇÃO SENOIDAL PARA ALGUNS VALORES DE $\beta$ E $\gamma=1$ E $1 / 7$.

\begin{tabular}{|c|c|c|c|c|c|}
\hline$S$ & $\beta=\pi$ & $\beta=3 \pi$ & $\beta=5 \pi$ & $\beta=7 \pi$ & $\beta=9 \pi$ \\
\hline$\gamma=1$ & 2.0894 & 5.0200 & 8.0878 & 11.189 & 14.305 \\
\hline$\gamma=1 / 7$ & 2.3293 & 5.3544 & 8.4066 & 11.486 & 14.581 \\
\hline
\end{tabular}

Como indicado anteriormente, um outro parâmetro geométrico de desempenho é a curvatura da modulação. Contudo, devido a complexidade de sua expressão analítica para a modulação senoidal, é mais interessante para efeitos de análise de desempenho apresentar apenas algumas de suas curvas, como mostra a Figura 10 para $\beta=3 \pi$ e $\gamma=1$ e $1 / 7$. Neste caso, observe que um valor de $\gamma$ menor do que 1 , diminui a curvatura da modulação, fato extremamente desejável para diminuir a perda de desempenho causada pelo erro de limiar.

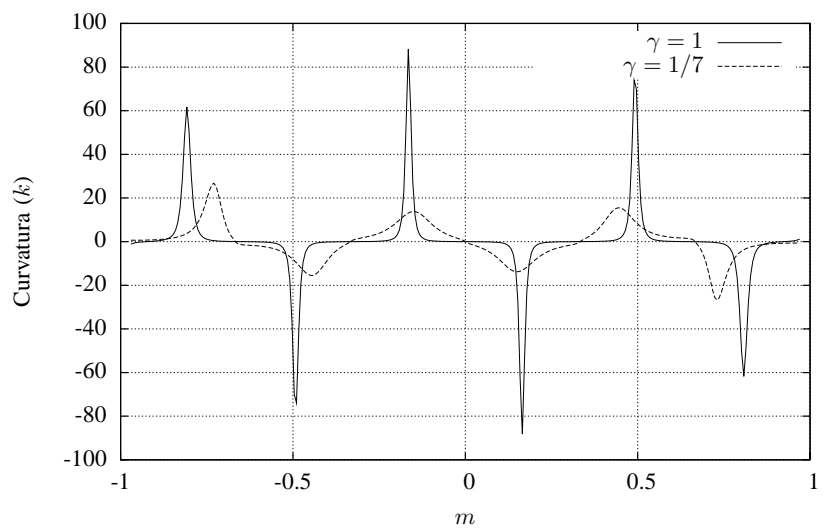

Fig. 10. Curvatura da modulação senoidal para $\beta=3 \pi$ e $\gamma=1$ e $1 / 7$.

O desempenho da modulação senoidal foi calculado por simulação computacional e comparado ao desempenho da modulação associada à curva espiral de Arquimedes considerada em [2] e em [9]. A curva espiral de Arquimedes uniforme é parametrizada por

$$
\mathbf{s}_{m}=A m\left[\cos \left(k_{p}|m|\right),-\sin \left(k_{p}|m|\right)\right],
$$

onde $k_{p}$ é uma constante que mede o desvio da modulação em relação à fase. A representação gráfica para essa modulação é mostrada na Figura 11 para alguns valores de $k_{p}$.

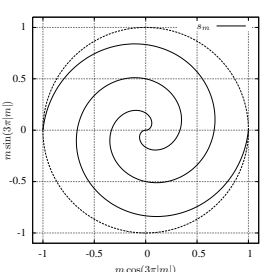

(a) $k_{p}=3 \pi$.

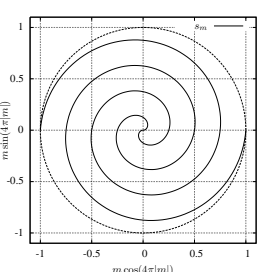

(b) $k_{p}=4 \pi$.

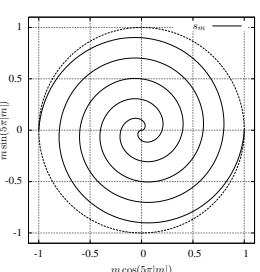

(c) $k_{p}=5 \pi$.
Fig. 11. Representação geométrica da modulação espiral de Arquimedes, para $k_{p}=\pi, 2 \pi, 3 \pi$ e $A=1$.

Neste caso, note que a ocorrência de erro de limiar na modulação espiral de Arquimedes, geralmente, implica em uma estimativa $\hat{m}$ de sinal contrário ao do valor enviado $m_{0}$, fato que normalmente não ocorre na modulação senoidal, pois o segmento da curva referente a $m>0$ está totalmente contida no semiplano $x>0$. Portanto, quando ocorre um erro de limiar na modulação senoidal, em geral o valor de $\left(\hat{m}-m_{0}\right)^{2}$ é menor do que o mesmo valor na modulação espiral de Arquimedes, fato que sugere um melhor desempenho da modulação senoidal em relação à modulação espiral de Arquimedes, quando a energia média de ruído não é suficientemente pequena. 
Comparando as curvas de desempenho das Figuras 12 e 13 podemos verificar que os pontos ótimos de desempenho da modulação senoidal são aproximadamente iguais aos apresentados na modulação espiral de Arquimedes. Contudo, analisando as curvas de desempenho da modulação senoidal, note que seus desempenhos abaixo dos pontos ótimos não caem tão rapidamente como na modulação espiral de Arquimedes. Tal fato está associado a influência do erro de limiar no $\overline{\epsilon^{2}}$, como explicado anteriormente.

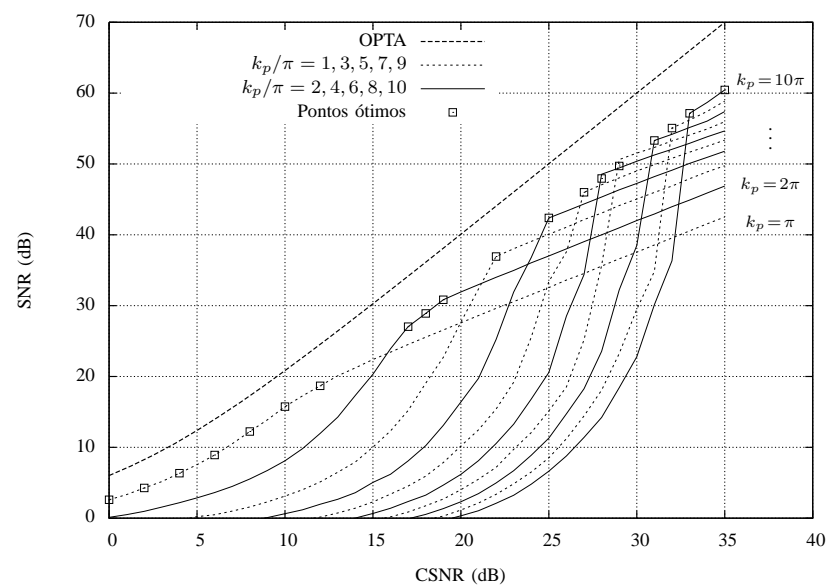

Fig. 12. Curvas de desempenho da modulação espiral de Arquimedes para $k_{p}=\pi, 2 \pi, \ldots, 10 \pi$.

A Figura 14 mostra as curvas ótimas de desempenho das modulações espiral de Arquimedes e senoidal com o objetivo de comparar seus desempenhos. Neste caso, o desempenho da modulação senoidal também foi calculado também para $\gamma=1$.

Neste momento, é importante chamar a atenção para o fato de que a diferença de desempenho entre as modulações com $\gamma=1$ e $\gamma=1 / 7$ ser decorrente, principalmente, da variação dos valores de suas curvaturas, como ilustra a Figura 10. Pois a diferença entre seus fatores de expansão não é significante, como indica a Tabela I. Este fato, novamente comprova a importância de se considerar a curvatura como um parâmetro relevante no projeto de modulações.

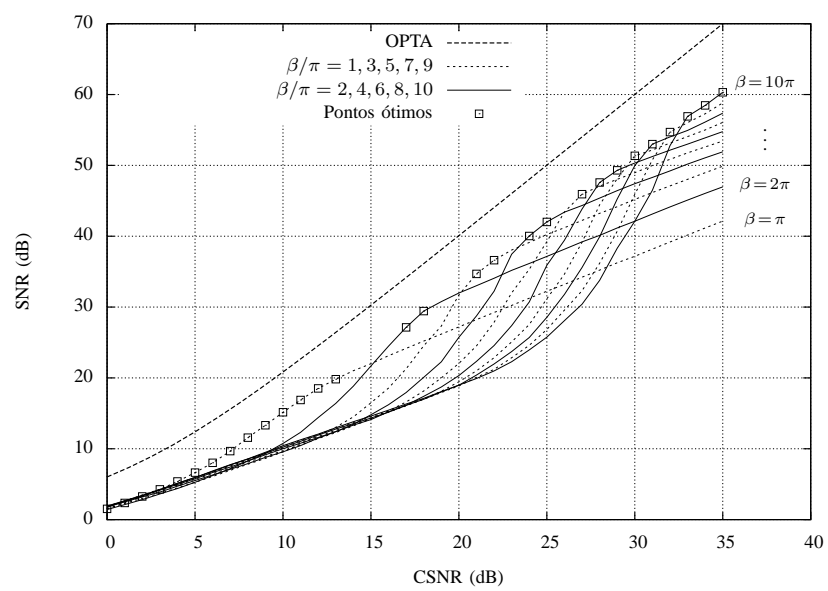

Fig. 13. Curvas de desempenho da modulação senoidal para $\beta=$ $\pi, 2 \pi, \ldots, 10 \pi$ e $\gamma=1 / 7$.

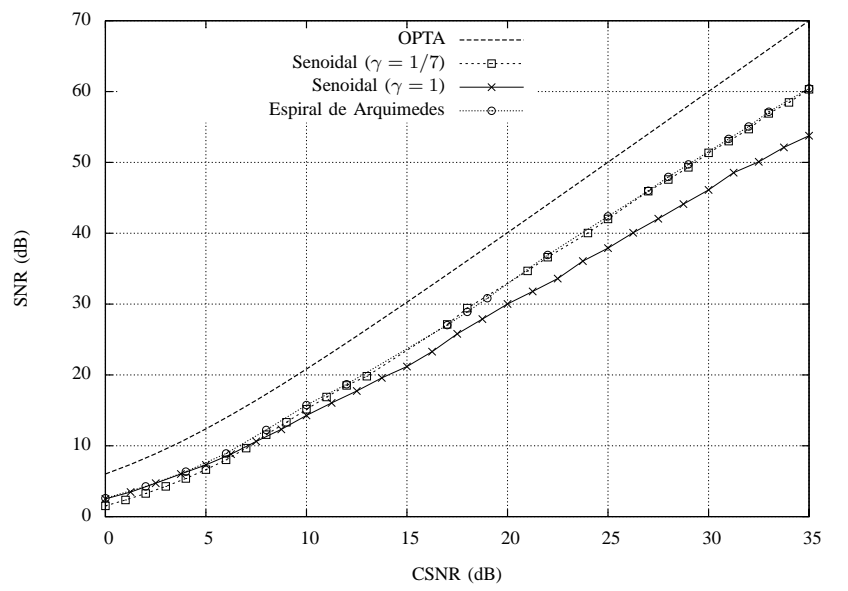

Fig. 14. Curvas de desempenhos ótimas das modulações espiral de Arquimedes e senoidal com $\gamma=1$ e $\gamma=1 / 7$.

\section{CONCLUSÕES}

A interpretação geométrica que associa modulações a curvas do espaço Euclidiano permitiu o uso de conceitos da geometria diferencial no contexto de projetos de modulações. Com isso, foi possível definir o conceito de curvatura de técnicas de modulação e verificar que esse parâmetro influencia no desempenho das modulações. Neste caso, uma condição entre a curvatura e a energia média de ruído foi derivada com o intuito de reduzir a ocorrência de erro de limiar. Além disso, um novo esquema de modulação não-linear foi proposto geometricamente, apresentando um bom desempenho e evidenciando a influência da curvatura no desempenho das modulações.

\section{AGRADECIMENTOS}

Os autores agradecem à FAPESP, ao CNPq e à CAPES pelo suporte financeiro recebido durante o período da realização desta pesquisa.

\section{REFERÊNCIAS}

[1] A.L. Garcia, Probability and Random Processes for Electrical Enginnering, Addison Wesley Longman, 2nd ed., 1994.

[2] C.M. Thomas, C. L. May and G. R. Welti, "Hybride amplitude-andphase modulation for analog data transmission," IEEE Transactions on Communications, vol. COM-23, no. 6, pp.634-645. June 1975.

[3] J.M. Wozencraft and I. M. Jacobs, Principles of Comunication Engineering, New York: John Wiley \& Sons, Inc, 1965.

[4] K.H. Lee and D. P. Petersen, "Optimal linear coding for vector channels,"IEEE Transactions on Communications, vol. COM-24, n0. 12, pp. 1283-1290, December 1976.

[5] M.P. do Carmo, Differential Geometry of Curves and Surfaces, PrenticeHall Inc., New Jersey, 1976.

[6] M.P. do Carmo, Riemannian Geometry 1nd ed. (Birkhäuser, Boston 1992).

[7] R.G. Cavalcante, Uma Análise da Influência da Curvatura do Espaço em Sistemas de Comunicações, Tese de Doutorado, FEEC-UNICAMP, Campinas, Brasil, 2008.

[8] R.G. Cavalcante, e R. Palazzo Jr. "Esquemas de modulação nãolineares associadas a superfícies mínimas," XXV Simpósio Brasileiro de Telecomunicações-SBrT’08, Rio de Janeiro, 2008.

[9] P.A. Floor, and T. A. Ramstad, "Noise analysis for dimension expanding mappings in souce-channel conding," IEEE - Seventh Workshop on Signal Processing Advances in Wireless Communications, Cannes, France, July, 2-5, 2006.

[10] S. Haykin, Sistemas de Comunicações: Analógicos e Digitais, Porto Alegre: Bookman, 4.ed., 2004. 